\title{
Predicting first episode psychosis in those at high risk for genetic or cognitive reasons
}

\author{
Stephen M. Lawrie*, Andrew Stanfield, Eve C. Johnstone and Andrew M. McIntosh \\ Division of Psychiatry, The University of Edinburgh, Kennedy Tower, Royal Edinburgh Hospital, Morningside Park, Edinburgh EH10 5HF, U.K.
}

\begin{abstract}
Structural and functional magnetic resonance imaging (MRI) of patients with psychosis has advanced to the point where there are clear abnormalities at a group level between patients and groups of healthy controls, and suggestions of different patterns of abnormalities between groups of patients. A major area of research endeavour is being able to translate these group differences into clinically relevant predictions at an individual patient level. Here, we briefly summarize our main findings in cohorts at high risk of psychosis because they come from families in which several members have schizophrenia or bipolar disorder, or have educational impairments. We highlight consistent predictors of psychosis in those at high risk of schizophrenia for genetic or cognitive reasons, as compared with quite distinct profiles between those at genetic high risk of schizophrenia $v$. bipolar disorder on functional MRI during an executive language task. We also consider future research directions and ethical issues in the early diagnostic testing of people at high risk of psychosis.
\end{abstract}

First published online 12 September 2012

\section{Introduction}

One of the greatest problems facing contemporary psychiatry is the lack of objective diagnostic tests. Brain imaging offers arguably the greatest potential in this regard, giving direct access as it does to the brain/mind in vivo. Magnetic resonance imaging (MRI) has particular advantages of wide availability and a lack of exposure to ionizing radiation. There have now been hundreds of structural and functional MRI studies of patients with schizophrenia and bipolar disorder as compared with controls which demonstrate consistent abnormalities in groups of the two patient populations (Lawrie \& Abukmeil, 1998, Wright et al. 2000). Direct and indirect comparisons demonstrate not only some overlap between the patient populations but also some possible distinctions. Patients with schizophrenia have reduced whole brain volumes, with greater reductions in prefrontal cortex and parts of the temporal lobe including the hippocampus and the amygdala; whereas patients with bipolar disorder tend to have similar abnormalities of a lesser degree, with possible sparing of some cortical regions and of the amygdala (Arnone et al. 2009). Here, we discuss whether these changes are evident in populations of high risk for schizophrenia or bipolar disorder, and the extent to which any such changes could be used to predict diagnosis at an earlier stage and perhaps in a more objective way than using standard clinical criteria.

* Address for correspondence: Stephen M. Lawrie, Division of Psychiatry, The University of Edinburgh, Kennedy Tower, Royal Edinburgh Hospital, Morningside Park, Edinburgh EH10 5HF, U.K.

(Email: S.Lawrie@ed.ac.uk)

\section{The Edinburgh high risk study (EHRS) of Schizophrenia}

The EHRS is a longitudinal prospective cohort study of people at high genetic risk of developing schizophrenia over subsequent years as they have at least two first and/or second degree relatives affected. Participants were aged 16-25 years at the beginning of the study (initial mean age 21 years) and recruited from all over Scotland. In total 162 such people gave some clinical, behavioural, cognitive and or brain imaging data, of whom 21 went on to develop schizophrenia (Johnstone et al. 2005). An additional 60 of the high risk subjects had psychotic symptoms, as elicited on a structured interview with the Present State Examination, which appeared to wax and wane in severity in association with cannabis use and life events, but did not translate into schizophrenia. Despite detailed cognitive testing the best performing neuropsychological test as a predictor of schizophrenia was actually a negative predictor, relatively good memory on the Rey-Auditory Verbal Learning Test being associated with the background level of risk for schizophrenia $(\sim 13 \%)$ in this population (Whyte et al. 2006). By far the highest positive predictive values (PPV) and negative predictive values (NPV) from nonimaging variables were two measures of schizotypy, the very detailed structured interview for Schizotypy (PPV 29\%, NPV 98\%), and the very quick selfcompleted Rust Inventory of Schizotypal Cognitions (PPV 50\%, NPV 94\%). Structural MRI scans at base line in 147 of the high-risk subjects showed that they had reduced volumes of the amygdala-hippocampal complex and thalamus compared with healthy controls (Lawrie et al. 1999), but these were relatively weak 
predictors of psychosis (Johnstone et al. 2005). Far more promising as a potential predictor with clinical utility was a measure of right pre-frontal cortex gyral folding the 'gyrification index' (GI) - which was increased in those subjects with high genetic risk who went on to develop schizophrenia compared with those who did not (PPV 64\%, NPV 58\%; Harris et al. 2007).

All the assessments were repeated at approximately 18-24 months and reductions in the left (para-) hippocampal uncus and fusiform gyrus characterized those with psychotic symptoms who went on to get schizophrenia - with similar predictive powers as above (Job et al. 2006). Adapting the GI software we developed (Moorhead et al. 2006) allowed us to automatically extract whole brain, prefrontal and temporal lobe volume across the entire 434 scans conducted over almost 10 years in the EHRS, despite the fact that we had to change the scanner approximately half way through. This technique showed reductions across all these brain regions of approximately 1-2\% per year until the time of diagnosis, on average 2.5 years later (McIntosh et al. 2011). Moreover, the extent of these reductions, particularly in the prefrontal lobe, was strongly and highly statistically significantly correlated with increasing severity of the symptoms that characterize psychosis, i.e. delusions, hallucinations and thought disorder.

Overall, in the EHRS, we have demonstrated that both changes over time in key brain regions, and even one base line structural MRI scan, appropriately analysed, can be used to predict schizophrenia years in advance on a single subject basis. We continue work to refine our image analysis protocols to increase the predictive power of our analyses.

\section{The Edinburgh study of comorbidity (ESC)}

The ESC has a very similar design to the EHRS, with the main differences being that we studied people at high risk of schizophrenia because they were educationally impaired (mean $\mathrm{IQ}=70$ ), and analysed the groups a priori as to whether they had in addition notable schizotypal features of a similar level to that in the EHRS which were predictive of schizophrenia. We hypothesized those at high risk because they had both low IQ and high schizotypy would exhibit what can be called 'an extended psychosis phenotype' that would put them at particularly high risk of developing psychotic symptoms and psychosis itself (Johnstone et al. 2007). This has indeed turned out to be the case. Low IQ subjects with schizotypy have very much increased prevalence of hallucinations and delusions (30-40\%), impaired memory and increased prefrontal cortical folding on the GI compared with those without
(Johnstone et al. 2007, Stanfield et al. 2008). Moreover, they also show a complementary pattern of reductions over time in grey matter in the left medial temporal lobe which relates to increasing psychotic symptoms (Moorhead et al. 2009). Early indications from ongoing analyses are that these changes over time are focused on the hippocampus, and are greater in those with psychotic symptoms including the small number who have gone on to develop schizophrenia per se.

In other words, it appears that participants in these two cohorts at high risk of schizophrenia for quite distinct reasons may have a common set of behavioural, cognitive and imaging predictors of schizophrenia (schizotypy, memory impairment and increased prefrontal gyral folding), which are particularly likely to translate into psychosis with grey matter loss over time in the medial temporal lobe.

\section{The Bipolar family study (BFS)}

A third prospective cohort study we have conducted in Edinburgh in recent years concerns those at high genetic risk of bipolar disorder because they have at least two close relatives who are affected with that condition. In total 112 high-risk subjects have participated, with an initial mean age again of 21 years at intake. We have only recently started to analyse and publish findings on this cohort, but our early findings suggest that they are manifestly different to those at high risk of schizophrenia for either genetic or cognitive reasons. Strikingly, during functional MRI while the subjects complete an executive language task (the Hayling Sentence Completion Test) there are quite distinct patterns of activation differences between the two genetic high-risk groups and healthy controls. In particular, during a parametric contrast in which increases or decreases in activation are mapped across varying levels of sentence constraint, thus controlling for non-specific activation in for example reading sentences and pressing buttons, subjects at high genetic risk of bipolar disorder show relative increases in amygdala activation compared with their group of matched healthy controls (Whalley et al. 2011). This is quite distinct from the pattern evident on studying those at a high genetic risk of schizophrenia, with exactly the same paradigm and contrast. Those people show a tendency to underactivate medial prefrontal cortex, thalamus and cerebellum (Whalley et al. 2004). Thus, it appears that functional MRI has at least the potential to distinguish populations at high genetic risk of different psychoses.

Functional MRI also appears to have the capability of differentially predicting depression and psychosis in both these populations. Of the subjects at high genetic risk for schizophrenia, those with psychotic symptoms showed a tendency to overactivate the parietal 
lobe during the Hayling Sentence Completion Task (Whalley et al. 2004) and this was a relatively strong predictor of schizophrenia when considered in combination with lingual gyrus activation in a post hoc analysis (Whalley et al. 2006). In 12 of those subjects who subsequently became depressed, to a clinically significant degree, reductions in dorso-lateral prefrontal cortex (right middle/superior frontal gyrus) as well as increased left superior temporal gyrus activation were evident, and these results survived controlling for the presence of positive psychotic symptoms at the time of the scan (Whalley et al. 2008). In marked contrast, those at high genetic risk of bipolar disorder who went on to develop a major depressive disorder, some of whom are likely to go on to develop bipolar disorder, have abnormalities in other regions that predict subsequent depression (Whalley et al. submitted).

\section{Ethical and practical considerations}

We describe here a set of findings in those at high genetic risk of schizophrenia that replicate in a cohort of those at high cognitive risk of schizophrenia and appear quite different in those at high genetic risk of bipolar disorder. There are also several indications that these results at least have the potential to be used in clinical practice to make early diagnoses of schizophrenia, and possibly also of depression in the context of psychosis. There are, however, some notable limitations of our own work and that from similar research groups that need to be acknowledged and several additional considerations before this work could be implemented into clinical practice.

\section{Representativeness}

One consideration is the extent to which our findings could generalize to those who are going on to develop schizophrenia or bipolar disorder in routine clinical practice. While it is true that the majority of patients are not at obviously high genetic or cognitive risk, because of a known family history or cognitive impairment, it is equally true that (apart from rare exceptions of individual genes of major effect) multiple genes contribute to the risk for schizophrenia and bipolar disorder and these are distributed throughout the population. Furthermore, there is at least as yet no indication that the biology of schizophrenia and bipolar disorder differs in those at high genetic or cognitive risk $v$. those who come from different backgrounds. In the only directly comparable data we are aware of, structural and functional MRI studies in populations at high risk of schizophrenia because they have psychotic symptoms show a very similar pattern of abnormalities in both those at high risk and in those who are going to make the transition to schizophrenia (i.e. are prodromal). Remarkably, reduced medial temporal lobe volume and increased prefrontal cortex volume are evident in those at high risk for clinical reasons, who go on to develop schizophrenia (Smieskova et al. 2010; Fusar-Poli et al. 2011).

\section{Clinical impact}

A greater concern is the extent to which the information we can derive with these measures can actually improve the clinical outcome for these people and do so in a cost effective way. It has to be acknowledged here that the best available evidence of the impact of reducing the duration of untreated psychosis is at best limited as to whether or not this improves subsequent outcome, whether by delaying the onset of schizophrenia or improving its subsequent prognosis (Marshall \& Rathbone, 2011). While some practitioners of early intervention are very enthusiastic (Bird et al. 2010), and such services may be cost effective (Knapp et al. 2011), it remains debatable whether any benefits accrue in routine clinical practice and in particular whether any additional benefits would be realized by introducing routine brain imaging in early psychosis. It is questionable whether a couple of hundred pounds for a structural MRI scan or several hundred pounds for a functional MRI scan would be cost effective in practice. On the other hand, the issues in schizophrenia and bipolar disorder are very similar to those in dementia, where structural or functional brain imaging has been recommended on a routine basis for several years. Initially, this was to exclude possible intracranial pathology (Foster et al. 1999), but in the past 10 years it has become clear that structural and functional brain imaging could have a role in early diagnosis and differential diagnosis of dementia. Indeed, the recent introduction of qualitative and/or quantitative changes into diagnostic criteria for minimal cognitive impairment and dementia, to facilitate clinical research, has recently been applauded (McKhann et al. 2011). An analogous situation would potentially ensue if we started employing brain imaging routinely in evaluation of first episode psychosis. The increasing realization that up to $10 \%$ of patients with psychosis have detectable auto immune encephalitides, which sometimes can be detected with MRI, provides further justification for such an approach.

\section{Practicalities}

We have shown in the EHRS that brain imaging measures are more powerful predictors of schizophrenia than clinical, behavioural and cognitive 
variables. Others have shown the same to be true in prodromal populations (see e.g. Koutsouleris et al. 2010, 2012). Structural and functional MRI also perform better than the best (and indeed only) genetic predictor in EHRS-the COMT val/met polymorphism (McIntosh et al. 2007). The most powerful prediction is however likely to come from a selective combination of a number of factors in these domains, as with most risk or outcome prediction models in medicine. This then raises issues about the anonymous storage and secure access to data of different types in a usable format. One can envisage a heavily populated database, preferably with data from several centres, to provide relevant reliable markers of what amounts to typical variation within control and psychiatric populations. In practice, it may be good enough to have varying ways of using imaging and other data across different centres depending on the amount of data that can be collected, much as in the recently proposed dementia criteria.

\section{Ethical issues}

Before any of these developments there is however a major need to address a number of ethical questions concerning the early diagnosis of psychosis. Very little work has thus far been done to address this. The availability of different treatments and knowledge of their effectiveness is likely to influence people's attitudes to early diagnostic testing and treatment. Indeed, acceptable rates of false positive and false negative diagnoses in different clinical scenarios would alter cut-offs, PPV and NPV for different decisions. The PPV and NPV quoted above in the EHRS are balanced for sensitivity and specificity because we have little information about which treatments would be acceptable. Qualitative studies and large population based questionnaire surveys are therefore required in various high risk populations to see at what level of risk of psychosis they might be prepared to take psychotherapeutic, behavioural or pharmacological interventions with known beneficial and adverse effects. It is for example possible that someone with relatively mild symptoms might be more prepared to accept screening than someone who might be at higher risk and with a greater potential to benefit from earlier treatment. In the rest of medicine, the uptake rate of cervical and breast cancer screening is encouraging, but that is for often fatal diseases for which there are increasingly effective treatments. As regards neuropsychiatric disorders, the uptake of Huntington's Chorea (HC) screening is much less successful, but there is of course no effective treatment for HC. Perhaps the most useful comparisons can again be made with reference to screening programmes for dementia, but that will be no substitute for work in those at elevated genetic, cognitive and clinical risk for psychosis.

\section{Conclusions}

We have briefly discussed here how it is possible to predict schizophrenia in those at elevated genetic risk and that it is possible to do so with high predictive power, particularly with brain imaging measures. The EHRS and ESC shows that a relatively small number of potent predictors identify an extended psychosis phenotype which might be of particular value in prediction. The Bipolar Family Study, in contrast, demonstrates quite a different pattern of abnormalities in those at high genetic risk of bipolar disorder as compared with the first two populations, in keeping with an ever increasing number of studies showing that schizophrenia and bipolar disorder can be distinguished with a number of brain imaging approaches. These may yet prove to be able to predict differential response to the types of treatment used in the two disorders, but this work remains to be done. There also remains a programme of work to translate what essentially remains as group profiles into individual risk scores, to find the optimal combination of imaging and other variables to enhance prediction power, to test the acceptability of these approaches in relevant populations, and then go on to demonstrate their practicality and cost effectiveness. This is a rather daunting agenda but brings with it the promise of impacting upon the outcome of psychosis as well as providing objective diagnostic and therapeutic markers in psychiatry.

\section{Declaration of Interest}

The research described in this editorial was funded by programme grants from the Medical Research Council (G9226254, G9825423, G0100102), the generosity of the Mortimer and Theresa Sackler Foundation, a Clinician Scientist Fellowship (Ref: 2268/4295) through the Health Foundation, and by NARSAD Independent Investigator and Senior Investigator Awards.

AMM is supported by a Scottish Funding Council Senior Clinical Fellowship. The investigators also acknowledge the financial support of National Health Service (NHS) Research Scotland, through the Scottish Mental Health Research Network (www. smhrn.org.uk) who provided assistance with subject recruitment and cognitive assessments.

SML and AMM have received financial support from Pfizer (formerly Wyeth) to conduct an imaging study of people with schizophrenia and bipolar disorder. SML, ECJ and AMM have done consultancy work for Roche Pharmaceuticals in connection with a 
possible new treatment for schizophrenia. AS has received a grant from Novartis for investigation of a new treatment for fragile $X$ syndrome. ECJ has done consultancy work for Novartis. SML has also received honoraria for lectures, chairing meetings and consultancy work from Janssen in connection with brain imaging and therapeutic initiatives for psychosis.

\section{References}

Arnone D, Cavanagh J, Gerber D, Lawrie S, Ebmeier K, McIntosh A (2009). Magnetic resonance imaging studies in bipolar disorder and schizophrenia: meta-analysis. British Journal of Psychiatry 195, 194-201.

Bird V, Premkumar P, Kendall T, Whittington C, Mitchell J, Kuipers E (2010). Early intervention services, cognitive behavioural therapy and family intervention in early psychosis: systematic review. British Journal of Psychiatry 197, 350-356.

Foster GR, Scott DA, Payne S (1999). The use of CT scanning in dementia. International Journal of Technology Assessment in Health Care 15, 406-423.

Fusar-Poli P, Borgwardt S, Crescini A, Deste G, Kempton MJ, Lawrie S, Mc Guire P, Sacchetti E (2011). Neuroanatomy of vulnerability to psychosis: a voxel-based meta-analysis. Neuroscience and Biobehavioral Reviews 35, 1175-1185.

Harris JM, Moorhead TWJ, Miller P, McIntosh AM, Bonnici HM, Owens DGC, Johnstone EC, Lawrie SM (2007). Increased prefrontal gyrification in a large high-risk cohort characterizes those who develop schizophrenia and reflects abnormal prefrontal development. Biological Psychiatry 62, 722-729.

Job DE, Whalley HC, McIntosh AM, Owens DG, Johnstone EC, Lawrie SM (2006). Grey matter changes can improve the prediction of schizophrenia in subjects at high risk. BMC Medicine 4, 29.

Johnstone EC, Ebmeier KP, Miller P, Owens DG, Lawrie SM (2005). Predicting schizophrenia: findings from the Edinburgh High-Risk Study. British Journal of Psychiatry 186, 18-25.

Johnstone EC, Owens DGC, Hoare P, Gaur S, Spencer MD, Harris J, Stanfield AW, Moffat V, Brearley N, Miller P (2007). Schizotypal cognitions as a predictor of psychopathology in adolescents with mild intellectual impairment. British Journal of Psychiatry 191, 484-492.

Knapp M, McDaid D, Parsonage M (2011). Mental health promotion and mental illness prevention: the economic case. Report published by the Department of Health, London.

Koutsouleris N, Gaser C, Bottlender R, Davatzikos C, Decker P, Jäger M, Schmitt G, Reiser M, Möller HJ, Meisenzahl EM (2010). Use of neuroanatomical pattern regression to predict the structural brain dynamics of vulnerability and transition to psychosis. Schizophrenia Research 123, 175-187.
Koutsouleris N, Gaser C, Patschurek-Kliche K, Scheuerecker J, Bottlender R, Decker P, Schmitt G, Reiser M, Möller HJ, Meisenzahl EM (2012). Multivariate patterns of brain-cognition associations relating to vulnerability and clinical outcome in the at-risk mental states for psychosis. Human Brain Mapping 33, 2104-2124.

Lawrie SM, Abukmeil SS (1998). Brain abnormality in schizophrenia. A systematic and quantitative review of volumetric magnetic resonance imaging studies. British Journal of Psychiatry 172, 110-120.

Lawrie SM, Whalley H, Kestelman JN, Abukmeil SS, Byrne M, Hodges A, Rimmington JE, Best JJK, Owens DGC, Johnstone EC (1999). Magnetic resonance imaging of brain in people at high risk of developing schizophrenia. Lancet 353, 30-33.

Marshall M, Rathbone J (2011). Early intervention for psychosis. Schizophrenia Bulletin 37, 1111-1114.

McIntosh AM, Baig BJ, Hall J, Job D, Whalley HC, Lymer GK, Moorhead TW, Owens DG, Miller P, Porteous D, Lawrie SM, Johnstone EC (2007). Relationship of catechol-O-methyltransferase variants to brain structure and function in a population at high risk of psychosis. Biological Psychiatry 61, 1127-34.

McIntosh AM, Owens DC, Moorhead WJ, Whalley HC, Stanfield AC, Hall J, Johnstone EC, Lawrie SM (2011). Longitudinal volume reductions in people at high genetic risk of schizophrenia as they develop psychosis. Biological Psychiatry 69, 953-8.

Mckhann GM, Knopman DS, Chertkow H, Hyman BT, Jack CR Jr, Kawas CH, Klunk WE, Koroshetz WJ, Manly JJ (2011). The diagnosis of dementia due to Alzheimer's disease: recommendations from the National Institute on Aging and the Alzheimer's Association workgroup. Alzheimer's and Dementia 7, 263-269.

Moorhead TWJ, Harris JM, Stanfield AC, Job DE, Best JJK, Johnstone EC, Lawrie SM (2006). Automated computation of the gyrification index in prefrontal lobes: methods and comparison with manual implementation. Neuroimage 31, 1560-1566.

Moorhead TWJ, Stanfield A, Spencer M, Hall J, McIntosh A, Owens DC, Lawrie S, Johnstone E (2009). Progressive temporal lobe grey matter loss in adolescents with schizotypal traits and mild intellectual impairment. Psychiatry Research: Neuroimaging 174, 105-109.

Smieskova R, Fusar-Poli P, Allen P, Bendfeldt K, Stieglitz R, Drewe J, Radue E, Mcguire P, Riecher-Rössler A, Borgwardt S (2010). Neuroimaging predictors of transition to psychosis - a systematic review and meta-analysis. Neuroscience and Biobehavioral Reviews 34, 1207-1222.

Stanfield AC, Moorhead TWJ, Harris JM, Owens DGC, Lawrie SM, Johnstone EC (2008) Increased right prefrontal cortical folding in adolescents at risk of schizophrenia for cognitive reasons. Biological Psychiatry 63, 80-85.

Whalley H, Mowatt L, Stanfield A, Hall J, Johnstone E, Lawrie S, McIntosh A (2008). Hypofrontality in subjects at high genetic risk of schizophrenia with depressive symptoms. Journal of Affective Disorders 109, 99-106. 
Whalley HC, Simonotto E, Flett S, Marshall I, Ebmeier K, Owens D, Goddard N, Johnstone E, Lawrie S (2004). fMRI correlates of state and trait effects in subjects at genetically enhanced risk of schizophrenia. Brain 127, 478-490.

Whalley HC, Simonotto E, Moorhead W, McIntosh A, Marshall I, Ebmeier KP, Owens DG, Goddard NH, Johnstone EC, Lawrie SM (2006). Functional imaging as a predictor of schizophrenia. Biological Psychiatry 60, 454-62.

Whalley HC, Sussmann JE, Chakirova G, Mukerjee P, Peel A, Mckirdy J, Hall J, Johnstone EC, Lawrie SM,
McIntosh AM (2011). The neural basis of familial risk and temperamental variation in individuals at high risk of bipolar disorder. Biological Psychiatry 70, 343-349.

Whyte MC, Brett C, Harrison LK, Byrne M, Miller P, Lawrie SM, Johnstone EC (2006). Neuropsychological performance over time in people at high risk of developing schizophrenia and controls. Biological Psychiatry 59, 730-739.

Wright IC, Rabe-Hesketh S, Woodruff PWR, David AS, Murray RM, Bullmore ET (2000). Meta-analysis of regional brain volumes in schizophrenia. American Journal of Psychiatry 157, 16-25. 